CIUDAD Y TERRITORIO

ESTUDIOS TERRITORIALES

ISSN(P): 1133-4762; ISSN(E): 2659-3254

Vol. LIII, № 207 , primavera 2021

Págs. 47-64

https://doi.org/10.37230/CyTET.2021.207.03

\title{
Evaluación de la efectividad en la regeneración urbana de nuestras ciudades. Lecciones aprendidas
}

\author{
Eduardo RUBIO-HUERTAS ${ }^{(1)}$ \\ José Må de Ureña-Francés ${ }^{(2)}$
}

(1)Ingeniero de Caminos, Canales y Puertos. Doctorando de la UCLM. (2) Profesor Emérito de Urbanismo y Ordenación del Territorio en la UCLM.

RESUMEN: Las experiencias de regeneración o rehabilitación urbana, ante la ausencia de evaluaciones concretas, han de ser programadas con previsiones de impactos medibles y cuantificables, garantizando el acierto de las decisiones. Se pretende establecer un marco actualizado de cómo se han evaluado las operaciones de regeneración urbana desde una perspectiva internacional, donde las secuelas de la crisis han hecho reflexionar sobre la necesidad de asegurar el cumplimiento de los objetivos. Se ha realizado una revisión bibliográfica de las experiencias en el mundo occidental, especialmente en España, identificando los factores determinantes, así como los casos de éxito.

PALABRAS CLAVE: Regeneración urbana; Rehabilitación urbana; Evaluación de la regeneración; Indicadores urbanos; Métodos de evaluación urbana.

\section{Evaluation of the effectiveness in the urban regeneration of our cities. Lessons learned}

\begin{abstract}
The urban regeneration actions must be programmed with predictions of measurable and quantifiable impacts, guaranteeing the correctness of decisions. It is intended to establish an updated framework of how urban regeneration operations have been evaluated from an international perspective, where the aftermath of the crisis has led to reflection on the need to ensure compliance with the objectives. A bibliographic review of the experiences in the western world, especially in Spain, has been carried out, identifying the determining factors, as well as the success cases.
\end{abstract}

KEYWORDS: Urban regeneration; Urban rehabilitation; Evaluation of regeneration; Urban indicators; Urban evaluation methods.

Recibido: 23.02.2020; Revisado: 08.10.20

Correo electrónico: erubioh@ciccp.es; № ORCID: https://orcid.org/0000-0002-5247-4326;

Correo electrónico: Josemaria.urena@uclm.es; № ORCID: https://orcid.org/0000-0002-0404-1454

Los autores agradecen los comentarios y sugerencias realizados por los evaluadores anónimos, que han contribuido a mejorar y enriquecer el manuscrito original. 


\section{Introducción}

E $\mathrm{n}$ las primeras dos décadas del siglo $\mathrm{XXI}$, se ha podido constatar una cierta tendencia hacia la globalidad como contrapunto a la amplia diversidad de metodologías, enfoques y criterios de análisis. Sería deseable que los sistemas de medida de sostenibilidad urbana, satisfacción con nuestros entornos, cumplimiento de expectativas y calidad de vida en general, pudiesen ser evaluables bajo patrones internacionales, estándares reconocidos y aceptados por todas las comunidades o países, que estuviesen basados en datos fáciles de obtener. Sin embargo, en la práctica de la regeneración urbana abundan la falta de uniformidad de criterios, el enfoque local, los intereses sectoriales y la secuencia temporal cortoplacista. Incluso desde el mundo académico y de la investigación, el análisis de la efectividad de las intervenciones de regeneración urbana puede llegar a ser tan dispar que se enfrentan teorías eminentemente sociológicas o humanísticas con visiones más aritméticas.

Ante este escenario, una postura pragmática puede ser la observación (crítica y analítica) de la experiencia, identificando no solo los casos de éxito, sino también los de menor impacto, con el fin de diseccionar los mecanismos de evaluación, tanto en la fase de concepción y diseño inicial de las iniciativas, como de la implementación de medidas, asociados a los programas y/o proyectos concretos de regeneración urbana. De este modo, se puede constatar qué planteamientos de partida son los más adecuados, cómo influye el marco teórico inicialmente propuesto, cómo se implementan los objetivos que se pretenden conseguir, qué instrumentos de gestión, control y seguimiento resultan más adecuados y qué agentes deben ser movilizados para la consecución de los objetivos deseados.

Pero este planteamiento no siempre resulta fácil de llevar a cabo, habida cuenta de numerosos factores que influyen en los procesos de regeneración urbana. Además, es una evidencia que existen tantas experiencias como modelos de gestión desarrollados (HERnÁndeZ-AJA \& MATESANZ, García \& Rodriguez, 2014) y, en consecuencia, cualquier pretensión de unificar criterios, indicadores numéricos $u$ otros elementos de ponderación para conseguir un automatismo para evaluar los resultados de los procesos de regeneración urbana, se torna una utopía.

Los aspectos determinantes sobre los que hoy en día se desarrollan las políticas de regeneración urbana son de carácter económico, social, físico y ambiental (PARKINSON, 2014), en una mezcla heterogénea con diferentes proporciones. El carácter de actuaciones integradas sugiere una evolución sobre enfoques tradicionales basados en políticas sectoriales que habitualmente se han venido desarrollando en las ciudades de forma independiente (p.e. rehabilitación de viviendas, mejora/reurbanización de espacios públicos, inclusión social, fomento de la actividad económica o mejora de la eficiencia energética de edificios e infraestructuras). La Regeneración Urbana Integrada (RUI) parte de la premisa de que la actuación de forma coordinada sobre áreas concretas produce sinergias positivas, por lo que se incluyen medidas que inciden sobre el espacio urbano, la calidad medioambiental, la promoción económica, la empleabilidad, la integración y bienestar social y la gobernanza comunitaria en la concepción y diseño de los programas y proyectos de regeneración urbana (NAVARRo \& RODRÍGUEZ, 2015a).

Por lo general, la mayor parte de las evaluaciones realizadas sobre las actuaciones de regeneración urbana son a posteriori y se centran en el grado de ejecución de las intervenciones, siendo mucho menor el número de evaluaciones de la efectividad de esas intervenciones en la calidad de vida de los habitantes y en la corrección de los aspectos que favorecieron la vulnerabilidad de esos barrios donde se ha actuado tras identificar la necesidad de su regeneración (HUUB \& BECKERSB, 2012).

El artículo presenta una síntesis de los factores que intervienen en la evaluación de los programas de regeneración urbana a partir de una revisión bibliográfica, especialmente en el ámbito anglosajón como referente en el inicio de los programas de regeneración integral y en el de España, como caso concreto.

\section{Evolución de la regeneración urbana desde mediados del siglo XX}

La magnitud del problema es bien distinta en algunas partes del mundo donde el reto en sí es, más que una "regeneración urbana", la propia "generación" estricta de asentamientos urbanos con unos mínimos de habitabilidad y dignidad. En Latinoamérica unos 90 millones son chabolistas (BANCo Mundial, 2019), más de 250 millones solo en Asia Oriental y Pacífico (BAKER \& GADIL, 2017) y en África casi 60 millones (SolEs I Coll, s.f.). Según datos de ONU Habitat, el porcentaje de población urbana que vivía en este tipo de asentamientos a nivel mundial en el año 2014 era del $29,8 \%$. 
Las realidades de países de la vieja Europa, como Francia con su problemática asociada a los "grandes ensambles" (GuerRand, 2004), Gran Bretaña y sus iniciativas como el New Deal for Communities Programme, pionero en el enfoque de intervenciones de regeneración basadas en áreas (LAWLESS, 2007), Italia o la apuesta por el reciclaje de su inmenso patrimonio arquitectónico (GASTALDI, 2017) o en los Países Bajos (Aalbers \& Van BeCKHoven, 2009), presentan diferencias en su evolución. Diferencias, tanto entre sí, como con los antiguos países socialistas donde el período de influencia soviética se caracterizó por el "urbanismo de bloques" (SAIZ, 2004), los escandinavos en su apuesta por la reinvención de los centros históricos (García-Hípola \& BeLtrán, 2011) o incluso los del Arco Mediterráneo, como es el caso de España y sus particularidades asociadas a cuatro décadas de dictadura.

Los procesos de regeneración urbana reglados, focalizados tanto en áreas concretas (barriadas), como en ciudades completas, han sido una constante desde mediados del siglo XX hasta la actualidad. Por lo general, en el denominado "mundo occidental"1, los enfoques han ido sufriendo transformaciones con el transcurso del tiempo en base a continuas adaptaciones, de acuerdo con los intereses de (algunos o diversos, pero no siempre todos) los agentes que intervienen en esos procesos complejos. Es habitual hablar de generaciones de políticas de regeneración, en referencia a períodos temporales con sus propias características económicas, sociales o políticas, con distintos protagonistas que implican políticas o maneras de afrontar los problemas identificados desde determinadas perspectivas (CARMON, 1999).

La denominada "era del bulldozer" fue una primera generación de políticas de intervención en las ciudades, puesta en marcha en la década de los 50, donde la recuperación del espacio físico degradado a través de intervenciones basadas en el saneamiento de los enclaves marginales de las ciudades mediante la sustitución de infraviviendas y el desplazamiento de sus habitantes, fueron la tónica habitual (SHORT, 1982), a través de actuaciones lideradas por las administraciones públicas (GiBSON \& LANGSTA, 1982). Es la etapa de la renovación (o reconstrucción).

Una segunda generación de regeneración urbana se manifestó en los años 70 y 80 , poniendo el énfasis en la etapa de la rehabilitación, tanto de viviendas como de los espacios públicos,

\footnotetext{
${ }^{1}$ A los efectos del presente artículo, se entiende como
} "mundo occidental" el formado por los países de tradición introduciendo el concepto de integralidad como elemento necesario para conseguir la mejora no solo del entorno físico, sino de sus habitantes, implementando acciones de carácter social e involucrando a la sociedad civil en los procesos a través de lo que se comenzó a llamar "participación ciudadana" (MOULAERT \& PARRA \& SWYNGEDOW, 2014). De esta época vienen fenómenos tan actuales como son los procesos de gentrificación (SMITH, 1979), por los que la población tradicional de los barrios es desplazada por nuevos residentes de mayor poder adquisitivo, generalmente tras un proceso de mejora y rehabilitación, tanto de las viviendas como del entorno físico (GRIFTH, 1996), aunque a veces también sucede por "contagio" de una zona adyacente a barrios de mayor poder adquisitivo.

La tercera generación de regeneración urbana comenzó a desarrollarse a principios de los años 80 , tras considerarse por parte de algunos gobiernos (fundamentalmente conservadores) en EEUU y en el Reino Unido el fracaso de los programas sociales de las décadas anteriores, desarrollándose una serie de alianzas entre los poderes públicos y privados, tanto a nivel individual, como con los agentes empresariales, inversores, comerciantes, etc. (Gibson \& Prathers, 1977).

Navarro (2015a) aporta la descripción de una nueva generación de tendencias en regeneración, con su origen a finales de los años 90 y principios del siglo XXI, la revitalización, donde el concepto de regeneración urbana integral se consolidó como marco de referencia para la programación de actuaciones, tanto a nivel de barrio como en ciudades completas. Las actuaciones van dirigidas a los espacios físicos y a las personas, potenciando las coaliciones o alianzas entre distintos agentes de los ámbitos privado y público, fomentando la participación inclusiva en órganos de gestión; es la época de las grandes intervenciones en el mundo occidental y sobre todo en Europa, asentada de forma global la importancia de las ciudades en el desarrollo socioeconómico de los estados. En esta generación, donde las ciudades, incluso los barrios, compiten entre sí para ser más atractivos de cara a los inversores y residentes potenciales, los proyectos culturales toman protagonismo como catalizadores de algunos procesos de revitalización de los barrios degradados, incorporándose al catálogo de medidas o herramientas habituales en los programas como impulso y valor añadido de los entornos (BIANCHINI \& PARKINSON, 1993; ApARICIO A., 2013).

anglosajona (y su traslación a los EEUU), así como los países europeos no procedentes del bloque soviético. 
La generación actual de políticas de regeneración urbana va más allá del concepto de integralidad y supera incluso los ámbitos de planificación tradicionales, es el tiempo de la reinvención. La cumbre de la Organización de las Naciones Unidas (ONU) celebrada en Nueva York el 25 de septiembre de 2015, marcó un punto de inflexión en la manera de entender diversos ámbitos de actuación a nivel de políticas de intervención, entre otros aspectos, sobre las ciudades. La aprobación de la Agenda denominada "Transformar nuestro mundo: la Agenda 2030 para el Desarrollo Sostenible" estableció los 17 Objetivos de Desarrollo Sostenible (ODS), entre los cuales cabe reseñar el Objetivo 11: Lograr que las ciudades y los asentamientos humanos sean inclusivos, seguros, resilientes y sostenibles, cuya primera meta, con un horizonte temporal establecido en 2030, consiste en:

"asegurar el acceso de todas las personas a viviendas y servicios básicos adecuados, seguros y asequibles y mejorar los barrios marginales" (ORganización de LAS NaCIONES UnidAs, 2015).

Con el vigente enfoque global de las actuaciones de regeneración, los ámbitos basados en áreas ceden paso a la creación de estrategias de ciudad, conectando incluso con cuestiones supramunicipales que abarcan más allá de las conurbaciones urbanas, compartiendo visiones con la Ordenación del Territorio como disciplina científica. En relación con el marco financiero de esta cuarta generación, PARKINSON (2014) afirma:

\footnotetext{
"Habrá que pasar de un modelo de desarrollo basado en las subvenciones públicas a otro en el que las asociaciones público-privadas, la innovadora ingeniería económica, una mayor cantidad de reciclaje de fondos mediante mecanismos destinados a tal fin con el sector público asuman mayores riesgos, pero también obtengan mayores recompensas". (p. 23).
}

En el caso concreto de España, y debido a las particularidades del conflicto civil y de la posterior implantación de un régimen totalitario centralista, la generaciones o políticas de recuperación de áreas urbanas han venido presentando ciertos desfases con el contexto europeo. Consecuentemente los procesos de renovación se generalizaron desde la década de los 60 hasta el final de la dictadura (en 1975), comenzando entonces la etapa de la rehabilitación hasta mediados de los 90, donde se inicia el predominio de la regeneración hasta el cambio de paradigma que supuso la crisis global inmobiliaria, para finalmente instaurarse la revitalización como práctica habitual en los programas e intervenciones (RUBIO \& UREÑA, 2020).

Tal como se representa en la FIG. 1, hay dos momentos/etapas adicionales relevantes: una etapa justo anterior a la desaparición del franquismo en la que las asociaciones de vecinos consiguieron bastante fuerza a la hora de plantear sus reivindicaciones y una etapa justo posterior a la dictadura, en la que se interviene solo en los elementos públicos de los barrios periféricos mediante reurbanización y re-equipamiento. Esta segunda etapa es muy evidente en cuanto hay ayuntamientos democráticos (muchos de ellos de ideología progresista) y ambas se producen ante todo en barrios periféricos (CERVERO-SÁNCHEZ, 2020). Son dos hechos relevantes porque el primero matiza la pura renovación (sustitución) y el segundo porque todavía no se interviene sobre los edificios lucrativos (vivienda y comercio en los barrios periféricos), si no solo sobre los espacios de acceso público (calles, parques, etc.), esto es sobre la urbanización y sobre los equipamientos.

Solo en la última década el impulso de la financiación europea ha favorecido la implementación del concepto de reinvención en forma de las Estrategias de Desarrollo Urbano Sostenible (EDUSIs), que abarcan conceptos más allá de la integrali-

\section{Contexto anglosajón y Europa Occidental}

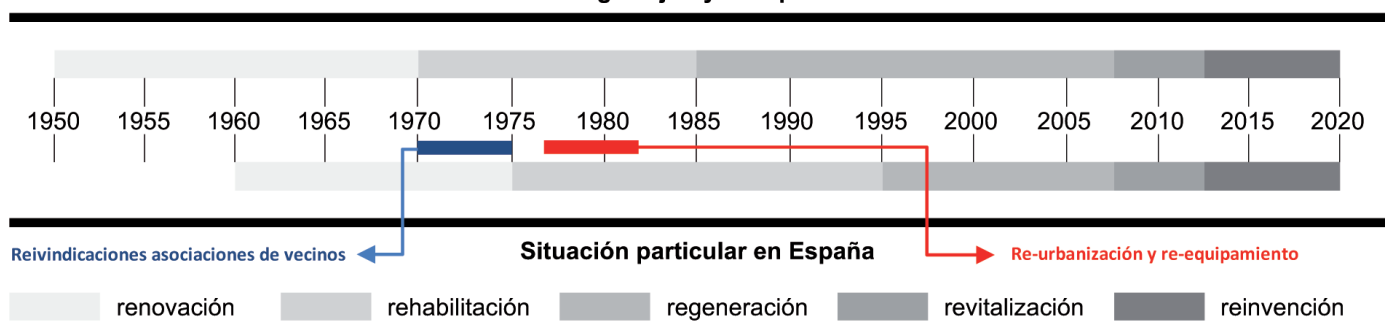

Evolución comparada de las políticas de mejora urbana.

Fuente: Elaboración propia basada en estudios previos de los autores. 


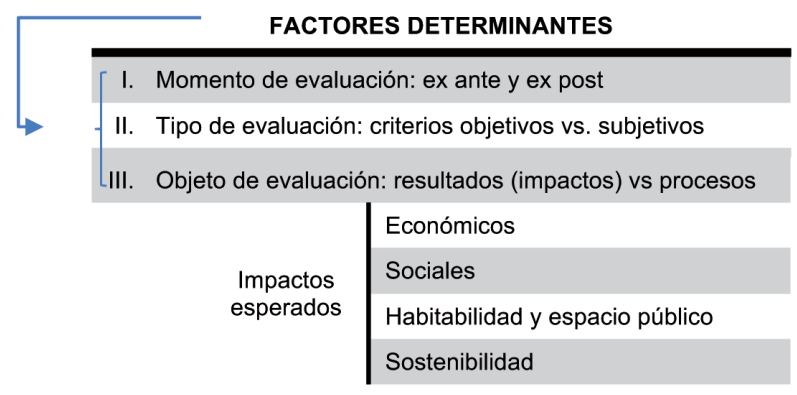

MÉTODOLOGÍAS DE EVALUACIÓN

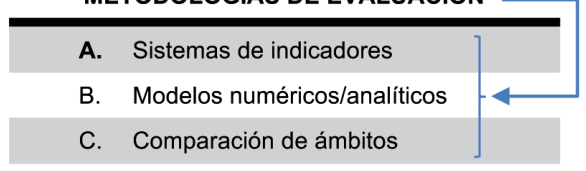

FIG. 2/ Resumen de factores y metodologías para la evaluación de programas de regeneración urbana.

Fuente: Elaboración propia basada en estudios previos de los autores.

dad, tal como se ha descrito anteriormente, incluyendo elementos como la sostenibilidad en toda su acepción.

\section{Aspectos clave para la evaluación de las consecuencias de las actuaciones de regeneración urbana}

A la hora de evaluar los impactos de las intervenciones o iniciativas de regeneración urbana basadas en áreas (Area-Based Interventions o ABls por sus siglas en inglés), la producción de definiciones, estudios, guías o tratados es tan amplia que costaría condensar solamente las tendencias, grupos de trabajo, evolución o líneas de investigación existentes al respecto, pues el tema se ha tratado desde innumerables puntos de vista, con enfoques que han variado a lo largo del tiempo en función de parámetros geoespaciales, socioeconómicos, e incluso bajo la continua influencia de los avances tecnológicos en lo relacionado con el tratamiento de la información, las posibilidades de la geolocalización o herramientas más avanzadas como la metodología BIM (Building Information Modeling), PSS (Planning Support Sytems), etc. Las secuelas de la última crisis han hecho reflexionar a las administraciones, como depositarios de la capacidad de decidir sobre el destino de las inversiones públicas, respecto de la necesidad de asegurar el cumplimiento de los objetivos marcados.

Se exponen a continuación (ver FIG. 2) una serie de factores que son determinantes en los procesos de regeneración urbana, y en base a los cuales suelen desarrollarse diversos métodos de evaluación de dichos procesos, pudiendo estar interrelacionados entre ellos, de acuerdo con los objetivos que predominan en el marco o políticas de referencia que rigen los distintos programas. Generalmente, en la práctica ni los factores, ni los métodos son excluyentes entre sí.

Las investigaciones sobre este tema concluyen que faltan herramientas prácticas y accesibles para comparar y seleccionar alternativas de intervención en la escala de barrio, incorporando criterios de sostenibilidad en el proceso de toma de decisiones (SIMÓN-RoJo \& HERNANDEZ-AJA, 2011), especialmente en lo que se refiere a aspectos sociales y económicos, de manera que se asegure la efectividad de las inversiones.

\subsection{Factores determinantes: momento de evaluación (ex ante y ex post)}

Si bien la metodología de evaluación ideal (utópica) es aquella que permite hacer previsiones fiables desde antes del inicio de los programas, incluyendo todo su ciclo de ejecución y sus consecuencias, como por ejemplo en los actuales programas de regeneración financiados por la Unión Europea, donde uno de los componentes en los documentos de diseño son los sistemas de indicadores y los valores esperados de mejora en un determinado período (es decir, una evaluación ex ante), la evidencia empírica es que frecuentemente se producen circunstancias imprevistas y/o sobrevenidas que alteran de forma más o menos significativa las condiciones de partida. Por ello, las evaluaciones a lo largo de los programas, incluso las que no están inicialmente previstas en su concepción, se hacen necesarias, siendo el de la comparación entre áreas una buena manera de llevarlas a cabo, con las limitaciones y reservas inherentes a dicha falta de planificación (MongIL, 2010). 
El ejemplo más significativo de evaluación ex post en España lo constituye el proyecto RUCOSA ${ }^{2}$ en el que se han analizado 81 programas de regeneración urbana (desarrollados en Andalucía entre los años 1990 a 2015) en base a tres elementos fundamentales: las condiciones de partida, el desarrollo del proyecto de intervención y sus impactos, de manera que se establecen una serie de escenarios evaluativos en función de la calidad sustantiva (diagnóstico, diseño, objetivos y medidas de los programas) y su lógica interna (coherencia entre las áreas de actuación y el diagnóstico, en los objetivos y en las actuaciones), generando diversos niveles de profundidad en el análisis y sus conclusiones (NAVARRO \& Rodríguez, 2015b).

\subsection{Factores determinantes: tipo de evaluación (criterios objetivos vs. subjetivos)}

No todo es "ciencia exacta" en relación con la evaluación de los programas de regeneración urbana, ni en su dimensión sustantiva (diagnóstico, objetivo y actuaciones), ni en su dimensión procedimental. Los análisis de satisfacción residencial evidencian la importancia de variables subjetivas interrelacionadas entre sí a la hora de valorar o considerar determinadas actuaciones que persiguen la complacencia de los residentes, pues se trata de identificar prioridades dentro de los programas para conseguir la mayor satisfacción (Von BREYMANN, 2014).

Desde el siglo pasado ya se ha venido manejando el concepto de indicadores de bienestar subjetivo (Subjective Well-Being o SWB por sus siglas en inglés) como alternativa a los clásicos indicadores sociales y económicos, ya que para entender el bienestar de una persona hay que medir las reacciones cognitivas y afectivas tanto respecto de su vida en general, como a diferentes aspectos de ella (DIENER \& SUCH, 1997). Resulta evidente pensar que una adecuada integración entre ambos enfoques puede aportar mejores resultados en los análisis tanto de diagnóstico, como de evaluación, pues no solo los SWB poseen una importante componente subjetiva, sino que el impacto de las medidas contenidas en los programas puede hacer cambiar la percepción en los residentes en función de si han sido directamente beneficiarios de alguna de ellas (NAVArRo, Rodríguez, Zapata \& MAteos, 2015a).

\footnotetext{
${ }^{2}$ Regeneración Urbana y Cohesión Social en Andalucía, financiado por el Fondo Europeo de Desarrollo Regional, en
}

\subsection{Factores determinantes: objetos de evaluación (resultado/impactos) vs. procesos}

A la hora de evaluar los programas, es importante considerar los objetivos previamente establecidos, enmarcados en el concepto de integrabilidad, porque dependiendo de ellos, las metodologías empleadas pueden variar sustancialmente.

\subsubsection{Impactos económicos}

Numerosos tipos de análisis inciden en los impactos económicos directamente asociados a las inversiones previstas (en fase de diagnóstico) o realizadas (en fase de ejecución). Estos análisis están muy presentes en los programas más actuales (Cervelló \& Segura, 2010; Morandi \& Pessina \& ScAvuzzo, 2010), habida cuenta de la participación del sector privado en los programas de regeneración que podrían denominarse de "cuarta generación", como se ha señalado anteriormente, y que por pura lógica han de poder estimarse con cierta precisión tanto las necesidades, como los resultados desde el punto de vista del rédito económico. Si bien tradicionalmente los resultados han primado sobre la propia gestión de los programas de regeneración urbana, en la actualidad la evaluación en términos de eficiencia de los recursos y los aspectos financieros están ganando protagonismo (JIMÉNEZ, MolinA \& NiCOLÁS, 2017).

\subsubsection{Impactos sociales}

La salud y el empleo se identifican como aspectos clave para una buena calidad de vida, factores ambos interrelacionados (en sus vertientes física y psíquica) e íntimamente relacionados con la vulnerabilidad y la desigualdad. La focalización de algunos programas de actuación en el empleo, en términos de acceso y de movilización social del individuo (NAVARRo, Rodríguez, ZAPATA \& MATEOS, 2015b), implica realizar evaluaciones específicas con metodologías analíticas y también empíricas.

En el caso de la salud, existen evidencias de su mejora para los residentes en áreas de regeneración urbana, debido a que la transversalidad de

el marco de la convocatoria de $1+D+i$ de la Consejería de Fomento de la Junta de Andalucía en 2013. 
las medidas implantadas repercute en los factores de riesgo que favorecen los niveles sanitarios en estas áreas (SERRANo \& al., 2014).

\subsubsection{Impactos en la habitabilidad y el espacio público}

La primera evidencia que se constata al evaluar de manera genérica los programas de actuación en áreas muy degradadas es que el factor catalizador de las mejoras en los barrios es la intervención sobre el espacio físico y su forma urbana. Esta hipótesis se sustenta en la génesis propia especialmente de los asentamientos "informales", donde la adaptación al medio es su fundamento y en donde las mínimas condiciones de acceso y conectividad constituyen la única forma de urbanidad en sus inicios, a partir de la cual su evolución y mejora va construyendo ciudad en la medida que los propios ocupantes del asentamiento disponen de más recursos.

Del mismo modo en las actuaciones sobre barrios planificados las medidas que antes son percibidas como positivas en términos cuantitativos e incluso cualitativos, son las que afectan al medio físico, tanto al espacio público (reurbanización, nuevas dotaciones) como a las ayudas directas para la rehabilitación o mejora de viviendas, (SÁNCHEZ DE MAdARIAGA, 2004).

\subsubsection{Impactos sobre la sostenibilidad}

En la actualidad, y al hilo de los 17 Objetivos de Desarrollo Sostenible, se han llevado a cabo metodologías para evaluar los procesos de regeneración urbana a partir de indicadores relacionados directamente con esos ODS (SÁNCHEZ DE MADARIAGA \& GARCIA \& SISTRO, 2018) de manera que faciliten la planificación coordinada con las agendas urbanas aplicables en razón de la ubicación del área (la FIG. 3 muestra un ejemplo).

\subsubsection{Medición de resultados vs. medición de procesos (gobernanza y participación ciudadana)}

Al margen de los impactos (o resultados), uno de los objetivos considerado clave como factor de éxito en los procesos de RUI, con especial incidencia en las actuaciones localizadas en barrios (las ya descritas ABIs), es la participación ciudadana, en cualquiera de las acepciones actuales: gobernanza, empoderamiento de la sociedad civil, etc. Los enfoques sectoriales tradicionales no tenían en cuenta a los residentes/usuarios ni se preveían órganos de control ni de gestión que incorporasen a representantes de los vecinos o sus asociaciones. En los años 80 fueron tomando fuerza los colectivos vecinales, impulsando e incluso liderando algunas actuaciones rehabilitadoras de relevancia (MANERo, 2010).

El impulso de la ciudadanía en la regeneración urbana en las iniciativas de los programas URBAN y similares de la Unión Europea (UE) ha evolucionado desde el nivel inicial de mera información/ constatación de las actuaciones, hasta la participación integral en todas las fases y órganos de decisión. Si bien las evaluaciones finales de los programas denotan que dichos procesos de participación ciudadana han quedado lejos de los objetivos perseguidos, lo cierto es que se ha

\begin{tabular}{lccc}
\hline Indicador & Unidades & Umbral verde & Umbral rojo \\
\hline Áreas verdes deportivas y de ocio & $\mathrm{m}^{2}$ per cápita & 27,55 & 6,67 \\
\hline Concentración de $\mathrm{NO}_{2}$ & $\mathrm{ug} / \mathrm{m}^{3}$ & 14,37 & 40 \\
\hline Concentración de $\mathrm{O}_{3}$ & días & 25 & 87,25 \\
\hline Concentración de partículas PM $_{10}$ & días & 3 & 35 \\
\hline Accesibilidad a la vivienda & $\%$ & 4,19 & 6,31 \\
\hline Resiliencia Urbana & índice & 50 & 10 \\
\hline Uso de transporte sostenible & $\%$ & 1 & 0,43 \\
\hline Vivienda protegida & \% sobre el total & 7,97 & 2,63 \\
\hline Vulnerabilidad urbana & \% sobre el total & 6,03 & 35,85 \\
\hline Accesibilidad a las áreas verdes urbanas & \% sobre el total & 23,88 & 4,99 \\
\hline Fig. 3/ Indicadores y umbrales estadísticos ODS 11 - Ciudades y comunidades sostenibles. & \\
\hline
\end{tabular}


instaurado una "manera de hacer" en la cual la gobernanza tiene un papel protagonista, estando presentes en la actualidad en cualquier proceso de RUI (DE Gregorio, 2013). Así, en la mayoría de los estados de la Europa Occidental se han implementado políticas en las ABls que implícitamente conducen a reconsiderar las formas de gobernanza local, aunque los mecanismos son diferentes en cada país, dependiendo de la tradición nacional respecto de los enfoques democráticos pluralistas (AtKInSON \& CARMICHAEL, 2011).

Muchas iniciativas de rehabilitación han sido conceptualmente bien diseñadas (diagnósticos acertados y medidas acordes al marco teórico) pero a la hora de su evaluación, tanto en la fase de implementación, como respecto de los resultados obtenidos, no se ha evaluado adecuadamente el papel de los habitantes y de los promotores (públicos y privados) en la dotación de viviendas de calidad, asequibles para los grupos de población con menos recursos (DWIJENDRA, 2013), evidenciando algunos fracasos motivados por la escasa participación de los actores o beneficiarios de dichos procesos. Esas situaciones se dieron fundamentalmente en las décadas finales del siglo pasado y ponen de manifiesto el problema existente cuando la práctica de la evaluación tiene lugar exclusivamente dentro de las estructuras de poder, por lo que la tendencia actual se basa en una evaluación participativa, con enfoques que abarcan desde los aspectos prácticos hasta los más intervencionistas que persiguen una auténtica transformación (TAYLOR \& BALLOCH, 2005). Políticas urbanas que fomentan la mezcla e integración de diferentes grupos sociales (las denominadas políticas de ingresos mixtos) mediante la producción de viviendas para los grupos sociales de ingresos más bajos (FRASER \& CHASKIN \& BAUZIN, 2013) también han puesto de manifiesto este fenómeno, pues el distanciamiento entre los planteamientos teóricos que rigen su diseño y los resultados que evidencian el paso del tiempo a medio y largo plazo (LUDWIG \& al., 2008) es notorio, al no contemplar aspectos como la toma en consideración de las distintas sensibilidades de los grupos sociales, tan dispares ente sí por su propia naturaleza.

En España los primeros procesos recientes de movilización y organización ciudadana por barrios se producen a finales de los años 1960's debido a la fuerte crisis urbana existente (carencia de servicios y equipamientos urbanos básicos, parque de viviendas inadecuado, etc.) y la falta de libertades políticas que permitiesen dialogar con los ayuntamientos, percibidos como ineficaces. La conquista más emblemática del derecho a la ciudad (LEFEBVRE, 1969) se produce en la vivienda y en la educación. Además, en el ámbito del urbanismo, pronto aparecen técnicos que de manera voluntaria o por afinidad ideológica, comienzan a apoyar con sus conocimientos la plasmación de las reivindicaciones vecinales, produciéndose los primeros contactos entre vecinos, técnicos y administraciones.

El final de los años 1970's y primera mitad de los 1980's fueron años de una profunda renovación de la cultura urbanística española. Las peculiaridades políticas de la transición determinaron que los nuevos planes hubieran de responder a los nuevos enfoques de calidad urbana y a las carencias infraestructurales elementales, que en otros países habían constituido la preocupación de generaciones de planes precedentes (EZquiaga, 2011).

En la primera etapa de ayuntamientos democráticos, la participación ciudadana se acrecentó,

\begin{abstract}
“...se hizo el mejor planeamiento (...) porque estuvieron representados en los municipios los movimientos... Veías en cada ayuntamiento a concejales que eran dirigentes vecinales, la época que vivieron como dirigentes vecinales les permitió transmitir las reivindicaciones que habían visto durante los últimos 15 o 20 años..." (Álvarez-MorA, 2020, págs. 164-165).
\end{abstract}

Pasados unos años, la participación se convirtió en un aspecto más bien formal. Solo más recientemente y vinculado a actuaciones urbanísticas de detalle, ha vuelto a aparecer como elemento clave para la asunción/empoderamiento de las actuaciones y para asegurar un buen apego para que las mejoras obtenidas sean cuidadas por los vecinos. Ejemplos como la gestión de la regeneración urbana del barrio de La Mina en San Adrián del Besós, en la que a pesar del intenso proceso de participación ciudadana (VELÁZQUEZ \& VeRdAGUeR, 2011), casi diez años después los vecinos seguían recelando de la intervención, indicando que no habían sido atendidas bastantes de sus demandas. En otros casos de reconfiguraciones de espacios púbicos problemáticos (calles, alamedas, plazas, etc.) también han destacado la relevancia de la participación ciudadana (RUIZ-APILÁNEZ \& UREÑA \& Solís, 2014).

\subsection{Metodologías: sistemas de indicadores}

Un indicador es:

"una variable o atributo relevante, determinado en función de su capacidad de medir los resultados de un objetivo a cumplir. Un dato estadístico, cuantificable o cualificable, de la realidad que puede verificarse a través del tiempo y proyectar su tendencia futura" (MARIANI, 2010, p.2) 
o también:

"una interpretación empírica de la realidad cuyas tres principales funciones son la simplificación, la cuantificación y la comunicación" (OCDE, 1997. p.1).

Los indicadores deben poseer la mayor precisión posible, pertinencia con el tema a analizar, sensibilidad a los cambios, fiabilidad y ser fácilmente obtenibles.

Analizando los estudios o metodologías referentes a la regeneración urbana (CusIDÓ, 2007; Agencia d'Ecología Urbana de Barcelona, 2011), se ha profundizado en los aspectos relacionados con la necesidad de medir, ponderar y prever las consecuencias de las actuaciones planteadas. El concepto de "sistemas de indicadores" está presente en la práctica totalidad de las referencias bibliográficas que describen herramientas, metodologías o simplemente parámetros medibles sobre la sostenibilidad urbana, la vida en las ciudades o la evaluación de los resultados obtenidos tras materializar programas de inversiones, de manera que pueden compararse distintos enfoques según las necesidades de información para las cuales se han diseñado, los ámbitos geo-políticos o espaciales para los cuales se han concebido, o incluso los parámetros de medición que pretenden determinar (RUEDA, 1999). Estos sistemas de indicadores son imprescindibles en la mayor parte de los procesos con concurrencia competitiva para acceder a la financiación de determinados programas de regeneración (p.e. el programa Europeo URBAN), constituyendo herramientas que permiten monitorear los objetivos perseguidos en los procesos de regeneración urbana (MARIANI, 2010), de manera que las conclusiones preliminares sirvan para un mejor enfoque de los problemas a afrontar, de las decisiones para programar inversiones y de la consecución de resultados.

Los sistemas de indicadores son frecuentes en los planes de recuperación del patrimonio urbano de los centros históricos, facilitando la participación ciudadana e interinstitucional en su gestión, simplificando la transparencia de la información en la identificación de las buenas prácticas, en la definición de los objetivos de planificación y en el seguimiento de los planes y proyectos (HugONY \& RocA, 2008). También son frecuentes en la evaluación de la Rehabilitación Urbana Integrada para ser analizados antes y después del plan o proyecto, para facilitar la comunicación entre los actores (vecinos, técnicos y administración), que al disponer de un modelo de análisis común puedan llegar a consensos sobre prioridades, para determinar causas y consecuencias de la elección de alguno de los elementos en detrimento de otros (HERNÁNDEZ \& al., 2015).
Las fuentes de los datos son diversas, pudiendo obtenerse información más genérica de bases de datos oficiales, con el nivel de desagregación del que se disponga -muchas veces no coincidente con la delimitación de las áreas intervenidas, con sus consiguientes inconvenientes- o bien mediante encuestas o entrevistas con los actores (Verdaguer, 2005).

La cantidad de información disponible es muy abundante, tanto como se quiera profundizar en los análisis concretos, por ello es determinante acotar los ámbitos temáticos, el tiempo y el marco espacial. En los estudios de diagnóstico de las ABIs, un primer grupo de indicadores clásicos se refieren al entorno (estado del espacio público, viviendas, servicios existentes), y por otro a las personas, en relación con su calidad de vida (demográficos, socioeconómicos, asociativos y de implicación en la vida pública) (Navarro \& ECHAVES \& MOYA, 2015a).

Los sistemas de indicadores relacionados con la sostenibilidad han sido habituales en las metodologías para la planificación, gestión y evaluación de la regeneración urbana desde principios del siglo XXI (VARGAS-YÁÑEZ, 2015). Los ciclos y procesos del metabolismo urbano en general y la rehabilitación edificatoria y del espacio público, han ido incorporando cada vez más peso ambiental. Bajo la denominación de "sustentabilidad" se han incorporado elementos integrados en los procesos que sean ambiental, social y económicamente adecuados para los habitantes actuales y venideros (BLANCO \& WAUtIEZ \& LLAVERO \& RIVEROS, 2001). Este enfoque de sostenibilidad adquiere especial relevancia en las grandes intervenciones, como por ejemplo, la estrategia de regeneración urbana denominada Urban Land Consolidation (ULC), empleada por el gobierno de Taiwan como herramienta para la renovación urbana con el objetivo de redensificar los espacios urbanos, donde la utilización de sistemas de indicadores como herramientas para la toma de decisiones de gran calado ambiental, social, político o económico se hace imprescindible (LIN, 2010).

A pesar de la incesante búsqueda de fórmulas repetitivas de aplicación directa, los indicadores denominados de $3^{a}$ generación tratan de poner la atención sobre el valor de lo local frente a la creciente globalización del enfoque de la sostenibilidad.

\footnotetext{
“...bajo ninguna argumentación científica o política puede ser homogeneizado el significado de la calidad de vida para todas y cada una de las ciudades del mundo. El derecho a la identidad, a la diferencia y a la diversidad no es negociable. Significa ello que la replicabilidad de las experiencias exitosas debe hacerse con responsabilidad" (LEón, 2013, p.179).
} 


\subsection{Metodologías: métodos numéricos/analíticos}

El análisis de la gran cantidad de información a procesar hace oportuno el empleo de modelos de evaluación de vulnerabilidad urbana (MEVU) basados en formulaciones matemáticas y estadísticas Salas \& Yepes, 2019a; (Bottero \& MondinI \& OpPIo, 2016). Los MEVU han de ser coherentes y compatibles con las herramientas empleadas para planificar estrategias de regeneración urbana, es decir, robustos frente a la incertidumbre, participativos como medio de incorporar las percepciones subjetivas de los interesados, consideradores de la naturaleza dinámica de vulnerabilidad urbana, abordadores de múltiples objetivos para mejorar la capacidad estratégica e implementadores de procesos cognitivos como forma de potenciar enfoques discursivos (SALAs \& YePES, 2019c). Se basan en complejos tratamientos estadísticos, métodos de análisis de decisión multicriterio cuando, además de varias alternativas, son distintos los factores de los que depende la decisión final, con una ponderación específica (FÉLIX \& RIONDET-COSTA \& PALMA-LimA, 2019).

Estas herramientas de planificación o decisión han sido desarrolladas en las últimas décadas y usadas en la implantación de infraestructuras o grandes dotaciones, donde los impactos económicos de la inversión y de los retornos esperados son fundamentales ${ }^{3}$.

En el caso de la RUI, las decisiones presupuestarias sobre planificación afectan a un espacio o área y la evolución de estas herramientas pasa ineludiblemente por la incardinación con sistemas de información geográfica (CERVERo \& AgustínHERnÁNDEZ, 2018), especialmente desde los años 90 (Lo \& Faber, 1997; Grande \& SECondinI \& VIRGILIO, 1999). Algunas herramientas complejas, basadas en estudios matemáticos/estadísticos, permiten evaluar de manera previa los escenarios de futuro que determinadas decisiones sobre infraestructuras vinculadas a los programas de regeneración urbana (SALAS \& YePES, 2019b).

\subsection{Metodologías: comparación de casos similares entre barrios intervenidos y no intervenidos}

Los programas y proyectos de regeneración urbana persiguen mejorar la calidad de vida en las áreas urbanas donde se desarrollan, por lo que la

\footnotetext{
${ }^{3}$ Un ejemplo de esta concomitancia son los modelos de priorización de áreas urbanas para proyectos integrados de revitalización y movilidad sostenible (Transit Oriented Development - TOD), como describen CAmpos-SÁnchez \& AbARCA-Álvarez
}

evaluación de su impacto tiene que comparar los cambios en la calidad de vida de los residentes respecto a su estado previo a la ejecución del proyecto, aunque esta comparación a veces no resulta fiable, puesto que la transformación puede que se deba a otras circunstancias distintas al proceso de regeneración urbana (RHODES \& TYLER \& BREnNAN, 2005). Un método de evaluación para asegurar que el cambio se origina en la intervención realizada es la comparación a lo largo del tiempo con lo que ocurre en otras áreas similares, donde no se han desarrollado intervenciones.

La metodología parte de la selección de ámbitos de comparación semejantes, esto es, con simimilares condiciones físicas y socioeconómicas. "Áreas experimentales", donde se han ejecutado programas de regeneración urbana integral y "áreas equivalentes", donde no ha habido intervenciones (al menos de carácter integral). Tanto las áreas experimentales, como las equivalentes, pueden haber sido beneficiarias de programas propios de las políticas sectoriales (vivienda, acción social, promoción económica, rehabilitación urbana, etc.). Es evidente que, a mayor número de casos de comparación, el análisis de las diferencias obtenidas mejora sustancialmente, aunque no menos importante es la metodología y condiciones empleadas en la elección y delimitación de esos pares semejantes y en su propia comparación. Básicamente, las áreas comparables han de presentar niveles similares de vulnerabilidad urbana en el momento previo a la puesta en marcha de las intervenciones de regeneración integrada en las áreas experimentales. Se han de definir una serie de criterios a la hora de establecer las áreas equivalentes respecto de una análoga experimental.

Al evaluar las diferencias obtenidas tras el análisis de los datos en relación con un período de tiempo determinado, conviene analizar en qué grado estas diferencias se deben a la aplicación de los programas de regeneración integral propiamente dichos o, por el contrario, obedecen a las diferencias en las condiciones de partida de las áreas comparables. Los indicadores o análisis que se realicen para comparar las áreas experimentales y semejantes pueden ser imprecisos o introducir posibles errores, debido a que los parámetros que se midan no tengan suficiente nivel de detalle o sean excesivamente genéricos desde los puntos de vista sectorial y espacial.

\& SeRRA-Coch \& ChAstel (2019), utilizados para facilitar la toma de decisiones a la hora de planificar inversiones asociadas a las infraestructuras del transporte, de gran calado económico. 


\section{Resultados de las evaluaciones: aciertos y fracasos}

Un recorrido por las actuaciones más relevantes de regeneración urbana y sus distintas evaluaciones, desde mediados hasta finales del pasado siglo, a través de los diferentes enfoques empleados para dar solución a los problemas planteados, muestra cómo se afrontan de distintas maneras en las ciudades problemas como la creación de guetos, la proliferación de la delincuencia o la deficiente movilidad. Se establece como prioridad la necesaria conveniencia de establecer unos objetivos en la fase de planificación que tengan en cuenta no solo factores propiamente asociados a la regeneración física de los espacios, sino también a sus consecuencias socioeconómicas (Segado-VÁzquez \& Espinosa-Muñoz, 2015).

Las experiencias con falta de evidencia de los resultados muestran que hay una serie de factores que las caracteriza. Se trata de una comprensión inadecuada de la teoría que subyace tras los problemas que provocan la situación de degradación de un barrio, el método seguido para su evaluación y una falta de información sobre el impacto de las intervenciones en los resultados clave que se han diseñado (RHOdEs, TYLER, \& BRENNAN, 2005; NAVARRO, 2015b).

Invertir en la rehabilitación del patrimonio arquitectónico, además de asegurar su protección en el tiempo, contribuye a la involución de ciertos procesos de degradación en centros históricos de las ciudades, poniendo en valor edificios como contenedores de actividades que pueden llegar a lideran los cambios de tendencias tanto desde el punto de vista físico o demográfico, como social y económico. Una adecuada planificación que vaya más allá de la dimensión local, con un enfoque territorial que tenga en cuenta la posición del área de intervención en la realidad geográfica de su entorno, puede favorecer la creación de centralidades que favorezcan la creación de oportunidades competitivas de los entornos intervenidos (Solis, UREÑA \& MoHÍNO, 2018). Para la evaluación de los resultados, conviene sin embargo no perder de vista los peligros de la excesiva especialización de estos centros históricos rehabilitados, y en concreto la prevalencia de los usos asociados al sector terciario, pues como ya puso de manifiesto TROITIÑO (1998):

\footnotetext{
"los responsables municipales y las administraciones turísticas deben ser conscientes de que convertir las ciudades históricas en «producto turístico», aun cuando esta función sea muy importante en la economía de su ciudad, implica asumir graves riesgos" (p. 213).
}

La diversidad de usos, así como el enfoque del espacio público como lugar de convivencia, sociabilización y motor de la vida en las ciudades, favorecen la conservación de los centros históricos frente a los procesos de abandono, degradación o incluso gentrificación. El concepto de sostenibilidad social (CALANTONIO \& DIXON, 2011), en un contexto de recesión económica ha evolucionado hacia el fomento de inversiones responsables y la responsabilidad social corporativa de inversores y promotores, donde las asociaciones público-privadas son frecuentes, incluso con la participación del denominado "cuarto sector" (organizaciones no gubernamentales), desarrollándose mecanismos adaptados a este nuevo escenario como convenios institucionales, herramientas de financiación o sistema de monitorización (estos últimos permiten la evaluación continua de los programas).

Algunas medidas para revitalizar los centros de las ciudades han sido favorecer la presencia de familias de clase media, sin hijos, o jóvenes profesionales, con viviendas en propiedad con el fin de paliar el contraste socioeconómico, pero la consecuencia inmediata ha sido el aumento de los precios, tanto de las viviendas, como de los alquileres y por tanto los procesos de gentrificación se han desencadenado de manera implacable (TELLOCAmpos \& Aguilar-Martínez, 2013), desplazando a los habitantes tradicionales. Iniciativas como la intervención pública favoreciendo la construcción o la rehabilitación de edificios para vivienda social dirigida a estos colectivos más vulnerables ayudan a equilibrar los efectos de las políticas de regeneración, aunque en estos casos los procedimientos de evaluación solo tienen sentido a posteriori y con intervalos temporales muy amplios.

Conviene no perder de vista los efectos colaterales asociados a determinadas intervenciones donde, después de procesos de regeneración urbana de barrios de vivienda social, se produce más gentrificación cuando la regeneración se ha hecho en barrios relativamente centrales y solo muy raramente se produce cuando se hace sobre barrios periféricos. La razón puede ser que en barrios centrales se facilita el contagio de barrios cercanos de clase alta y en la periferia es menos posible (Leal, 2004; Leal \& Sorando, 2013).

Otras experiencias basadas en el contacto íntimo y directo con los residentes y usuarios de los barrios afectados han reportado resultados positivos mediante la adopción de un enfoque antropológico, el denominado "urbanismo participativo" (CASTRILLO, 2014), constatando, al igual que sucede con las iniciativas URBAN, una mejora en la implantación 
de una cultura de la participación de la sociedad y de escucha por parte de las administraciones. En estos casos, la evidencia de los resultados se constata en el nivel de satisfacción de los propios residentes como partícipes de los procesos.

Experiencias innovadoras de autofinanciación de las actuaciones por parte de los residentes, más allá de la mera rehabilitación de viviendas, mediante procesos de economía local como han supuesto las cooperativas vecinales (RosA, MÁRQUEZ \& NAVAS, 2017), son fácilmente evaluables ya que la implicación de los vecinos posibilita el acceso a la información sobre los datos de resultados, tanto desde el punto de vista económico (inversión/capitalización), como de la satisfacción o en lo relativo a aspectos sociales (integración, empleabilidad, identidad barrial).

Un factor determinante para lograr el éxito de los programas de intervención es la implicación de los profesionales encargados de la gestión de los procesos en todas sus fases, la perspectiva bottom-up o "desde abajo" en castellano (Navarro, 2015c). Por su carácter de integrabilidad, los distintos ámbitos de gestión involucrados en los procesos de regeneración urbana precisan del personal especializado en materias como la planificación territorial, las infraestructuras, la promoción económica y cultural, la educación y los servicios sociales o incluso la seguridad. En consecuencia, cuanto más estrecho sea el grado de participación en los procesos de concepción y definición de actuaciones, mayor será la implicación de los responsables de su materialización y gestión en el día a día de su implantación, así como en los procesos de evaluación.

Un elemento central habitual en los esquemas que buscan la regeneración integral es la concentración en los primeros años de la iniciativa de actuaciones para mejorar el entorno físico de la zona en cuestión. Este aspecto se ha identificado como importante no solo como una forma de mejorar la apreciación de los residentes como de los posibles nuevos vecinos, sino también como un elemento clave de la estrategia diseñada para implantar nuevas actividades (RHODES \& TYLER \& BRENNAN, 2005). En definitiva, la mejora de los aspectos físicos (vivienda, urbanización, etc.) es mucho más rápida que la mejora de los aspectos sociales y económicos (empleo-paro, formación, integración social). Los primeros pueden hacerse en 5-10 años y los segundos requieren al menos 30 años (una generación). Por ello las actuaciones socioeconómicas deben mantenerse mucho más tiempo que las físicas. Este decalaje temporal en las actuaciones presenta dificultades a la hora de evaluar los procesos, poniendo de manifiesto la conveniencia de programar actividades que se mantengan en el tiempo.

En la gran mayoría de los procesos de regeneración urbana identificados como "casos de éxito" no cambian el modelo urbano o morfología del barrio (p.e. se mantiene el modelo de bloque abierto cuando esa era la situación previa) y solo en muy pocos casos se cambia dicho modelo, cuando se compagina rehabilitación de edificios y tejidos con derribo y reconstrucción de partes del barrio. Ejemplos de esta combinación son la Unidad Vecinal de Absorción "UVA" de Fuencarral en Madrid, el barrio de la Mina en Sant Adrià de Besòs y su enfoque integral innovador (JORNET \& LLOP \& PASTOR, 2008), el barrio de Bijlmermeer en Amsterdam o el barrio de Regent Park en Toronto.

La flexibilidad debe ser una condición determinante en las políticas de regeneración urbana, de manera que los diferentes actores intervengan en cada fase del proceso, desde la percepción de los problemas a solucionar, hasta la construcción de estrategias que relejen su percepción de esos problemas bajo un enfoque basado en evidencias y el conocimiento de lo que funciona, todo ello en un marco que permita adaptarse a las circunstancias en el tiempo. Esto último es importante en tanto en cuanto los aspectos sociales y laborales de los programas de regeneración urbana son los que arrojan menos resultados positivos y además requieren la continuidad de las actuaciones durante más años.

Algunos ejemplos en el contexto occidental de cómo enfocar la evaluación de los programas de regeneración urbana pueden ser los Empowerment Zones en EEUU utilizando mecanismos cruzados (internos y externos al programa) basados en indicadores que se repiten a lo largo del tiempo, los Hommes and Communities en Reino Unido centrados en análisis coste-beneficio o la Politique de Ville en Francia a través del Observatorio Nacional sobre las Zonas Urbanas Sensibles creado por Ley y que actúa mediante la evaluación sistemática de indicadores previamente establecidos (APARIcIO \& DI NANI, 2011).

\section{Discusión y conclusiones}

Tal como se constata en la bibliografía consultada, las evaluaciones de los programas de 
regeneración urbana han tendido hacia la eficacia frente a la efectividad, es decir, se han centrado sobre el cumplimiento de los aspectos instrumentales más que en asegurar que las actuaciones resuelven las causas que originan la vulnerabilidad. Los factores que intervienen en los procesos de regeneración integral de áreas urbanas son variados, complejos y diferentes entre sí, por lo que una correcta evaluación pasa por el análisis en fases previas, en las de implementación y en posteriores a la finalización de las actuaciones.

Han sido diversas las maneras de evaluar dichas intervenciones, desde metodologías basadas en criterios subjetivos, sistemas de indicadores más o menos complejos, procesos de gobernanza y participación ciudadana, hasta otras que miden los impactos económicos de las actuaciones en las zonas intervenidas o incluso las que estudian la comparación de casos similares intervenidos y no intervenidos.

Como se ha explicado anteriormente, los análisis de satisfacción se basan en componentes fuertemente subjetivas y por tanto su estandarización es compleja. Si bien la información necesaria para la evaluación ha de proceder de encuestas a residentes, distinguiendo entre aquellos que han sido directamente beneficiarios de medidas específicas de los programas de actuación y los que no lo han sido (NAVARRO \& RodríGUEZ, 2015b; NAvARro \& Rodríguez \& Zapata \& MAteos, 2015a), los resultados en dichas evaluaciones pueden no ser tan representativos de la realidad como pudiera esperarse. Los efectos de los procesos de mejora en la calidad de vida de los residentes, de acuerdo a determinadas estrategias de intervención, no pueden sino arrojar resultados a medio o incluso largo plazo, por lo que el análisis de informaciones obtenidas en base a experiencias puntuales y/o localizadas en el tiempo no parece el más adecuado. Del mismo modo, es evidente que la mera expectativa de que exista una continuidad en la intervención sobre los aspectos que confieren el carácter de vulnerabilidad a las áreas intervenidas (y su tutela por parte de los gestores de los programas) confiere por sí misma una cierta sensación positiva entre los residentes, frente a la sensación de desamparo que impregnan actuaciones en sentido contrario.

Los sistemas de indicadores como herramienta para la evaluación de planes o programas de rehabilitación han evolucionado en el último medio siglo acorde con los cambios en los enfoques del concepto de regeneración urbana propiamente dicho y con la incorporación de criterios de sostenibilidad, integrabilidad o gobernanza. Sin embargo, la evaluación de estos programas presenta dificultades por la precisión temática y espacial de la información disponible para referirse a los temas concretos que pretende la regeneración, pues es posible que no haya datos o indicadores estadísticos específicamente para dichos temas o zonas sobre los que se actúa; quizás haya datos o indicadores estadísticos desagregados, pero que no coincidan con la delimitación de los barrios intervenidos, por lo que los datos no son precisos espacialmente para medir lo que ha sucedido en esos barrios.

Tal como sugiere LEÓN (2013), la evaluación de los procesos de regeneración basada en indicadores ha de descender al nivel de lo local, pues cada modelo de sociedad tiene sus propios estándares de calidad de vida. Pero incluso en una misma ciudad, cada barrio, por lo general coincidente con una determinada área de intervención, presenta su propia idiosincrasia que debería ser tenida en cuenta a la hora de establecer los sistemas de indicadores a considerar para diagnosticar sus problemas, para establecer las medidas para paliarlos y para evaluar la efectividad de las actuaciones realizadas.

Enfoques sofisticados, con tratamientos estadísticos modelizados de la información obtenida procedente de la gran cantidad de datos (aunque a veces no lo suficientemente precisa), que facilitan las tecnologías digitales, si bien aporta un plus de mejora en cuanto a su fiabilidad, minimiza la aplicabilidad de las herramientas por entidades o administraciones no siempre dotadas de los medios necesarios para su procesamiento.

Evaluar la efectividad de la regeneración urbana en base al grado alcanzado de participación ciudadana y gobernanza, a pesar de que es una práctica que se ha venido generalizando en las dos últimas décadas, arroja resultados un tanto decepcionantes en lo que a implementación de la planificación colectiva se refiere, si bien muestran una cierta progresión en cuanto a lo que DE GREGorio (2013) denomina "manera de hacer las cosas".

Sin embargo, conviene no perder de vista los problemas que la participación pública mal entendida puede originar. Situaciones como la deriva asamblearia, donde la efectividad se pierde por la discusión de los aspectos más intrascendentes 
de las decisiones a tomar, o la intromisión de decisores ajenos a los verdaderamente implicados que se instauran casi de manera profesionalizada en los foros de discusión y participación, e incluso personas o colectivos interesados en cuestiones meramente particulares (o sectoriales) que pueden llegar a condicionar la gestión de los programas. Este enfoque crítico no es novedoso, pues la bibliografía ya lo evidencia, incluso en la sociedad británica, considerada pionera de los enfoques participativos en programas considerados de referencia como el City Challenge (DALGLEISH, 1996).

En cuanto a la evaluación de los resultados de la RUI a partir de factores económicos, el giro de las políticas de un marcado social durante las décadas de los 60 y 70 (LAWLESS \& BROWN, 1986) hacia posiciones más neoliberales, sobre todo impulsadas por los gobiernos conservadores de EEUU y Gran Bretaña, favoreció la presencia del capital privado como agente llamado a liderar los procesos de cambio y mejora de la ciudad degradada (NABARRO, 1980).

Es de esperar que, para esos agentes empresariales, la eficiencia de los procesos de regeneración sea evaluable en términos de impactos económicos. Pero este enfoque a veces dista de los planteamientos de mejora social, equidad y sostenibilidad que impregnan el carácter integral de los programas. Por este motivo las metodologías de evaluación basadas en factores económicos no pueden ser sino complementarias de otros procesos globales de análisis de resultados, constituyendo los aspectos relacionados con el incremento de las tasas de actividad, la revalorización de inmuebles (viviendas y locales) o el aumento del precio de los alquileres, factores a tener en cuenta como indicadores de buenos resultados, pero solo en el marco de unas previsiones donde otra serie de aspectos globales puedan tener como consecuencia los anteriores, y por supuesto siempre dentro de los límites previstos compatibles con la mejora de la calidad de vida de los residentes.

La comparación de barrios en los que se ha intervenido con otros similares no intervenidos como método de evaluación de la eficiencia de las medidas desarrolladas presenta no pocas dificultades en la práctica. Desde la propia delimitación y selección de las áreas comparables (NAVARRO, ECHAVES \& MOYA, 2015b) dadas las peculiaridades y singularidades de cada barriada y sus habitantes, las dificultades para conseguir "testigos" con similares parámetros de población, localización, condiciones físicas o situación socioeconómica, hasta la propia manera de discernir si las mejoras en las condiciones de vida de los residentes son consecuencia de los impactos que los programas de regeneración urbana han conseguido (NAvarRo \& Guerrero \& Moya, 2015) frente a otras consecuencia propias del contexto general de la ciudad o incluso de acciones inherentes a las tradicionales políticas sectoriales que actúan de forma continuada.

\section{Bibliografía y referencias}

Aalbers, M. \& Van Beckhoven, E. (2009): The integrated approach in neighbourhood renewal: more than just a philosophy?, Tijdschrift voor Economische. Sociale Geografie: Vol. 101, No. 4, 449-461.

Agencia d'Ecología Urbana de Barcelona (2011): Sistema de indicadores y condicionantes para ciudades grandes y medianas. Barcelona (España): Ministerio de Fomento.

Álvarez-Mora, A. (2020): MAET 1 Ciudad. Jornada de Debate sobre las Relaciones entre Proyecto Arquitectónico, Proyecto Urbano y Plan de Ordenación Urbana, (págs. 164-165).

Aparicio, A. (2013): Los centros históricos de Toledo y Cuenca. Nuevos equipamientos socioculturales para la recuperación funcional. Polígonos. Revista de Geografía, 25, 19-56.

- \& DI NANI, R. (2011): Modelos de gestión de la Regeneración Urbana. Madrid (España): SEPES Sociedad Estatal de Suelo.

AtKInson, R. \& CARMichael, L. (2011): Neighbourhood as a new focus for action in the urban policies of West European states. En I. SMITH \& E. LEPINE \& M. TAYLOR (Edits.), Disadvantaged by where you live? London, United Kingdom: Policy Press.

BAKER, J. \& GADIL, G. (2017): Obtenido de http://hdl.handle.net/10986/27614

Banco Mundial (2019): Informe anual 2019. Poner fin a la pobreza, invertir para generar oportunidades. Obtenido de https://www.bancomundial.org/es/ about/annual-report

BiANCHINI, F. \& PARKINSON, M. (1993): Cultural Policy and Urban Regeneration: The West European Experience. Manchester, United Kingdom: Manchester University Press.

Blanco, H. \& Wautiez, F. \& Llavero, A. \& Riveros, C. (2001): Indicadores regionales de desarrollo sustentable en Chile: ¿Hasta qué punto son útiles y necesarios? EURE, Volumen XXVII, $n^{\circ}$ 81, 85-95.

BotTero, M. \& MondinI, G. \& Oppio, A. (2016): Decision Support System for evaluating urban regeneration. Procedia-Social and Behavioral Sciences, 223, 923-928. 
Calantonio, A. \& Dixon, T. (2011): Urban Regeneration and Social Sustainability. London (United Kingdom): Wiley-Blackwell.

Campos-Sánchez, F. \& Abarca-Álvarez, F. \& Serra-Coch, G. \& ChAstel, C. (2019): Evaluación comparativa del nivel de Desarrollo Orientado al Transporte (DOT) en torno a nodos de transporte de grandes ciudades: métodos complementarios de ayuda a la decisión. EURE, Volumen 45, $n^{\circ} 134,5-29$.

CARMON, N. (1999): Three generations of urban renewal policies: analysis and policy implications. Geoforum, 30, 145-158.

CAStRILlo, M. (2014): ¿Urbanistas «en transición»? El enfoque antropológico del mejoramiento urbano y sus efectos sobre la concepción urbanística. Ciudad y Territorio. Estudios Territoriales, Volumen XLVI, $n^{\circ}$ 179, 81-97.

Cervelló, R. \& Segura, B. (2010): Un modelo para evaluar y optimizar el impacto espacial de las inversiones en regeneración urbana. Investigaciones Regionales, 17, 125-138.

Cervero, N. \& Agustin-Hernández, L. (2018): Evaluación gráfica de la rehabilitación urbana del poblado dirigido de Caño Roto. ACE - Arquitectura, Ciudad y Entorno, Año 13, núm. 37, 155-182.

Cervero-SÁnchez, N. (2020): La inciativa ciudadana, como origen del cambio urbano. La remodelación del Poblado Dirigido de Orcasitas en Madrid. Ciudad y Territorio Estudios Territoriales, $n^{\circ} 204$, 277-294.

Cusıdó, O. (2007): Herramientas para la evaluación continua: Observatorio e indicadores de seguimiento. En X. Casanovas, Método RehabiMed Arquitectura Tradicional Mediterránea I. Rehabilitación Ciudad y Territorio. Barcelona (España): Collegi d'Aparelladors i Arquitectes Tècnics de Barcelona para el consorcio RehabiMed. Obtenido de http://www.rehabimed.net/es/2015/11/metodo-rehabimed-arquitectura-tradicional-mediterranea-irehabilitacion-ciudad-y-territorio/

DaLgLeISH, K. (1996): An analysis of the City Challenge decision-making process: The Dearne Valley Partnership and Sheffield. Masters (United Kingdom): Sheffield Hallam University.

DE Gregorio, S. (2013): La Iniciativa Comunitaria URBAN como factor de transformación de la práctica de la regeneración urbana: aproximación al caso español. Ciudad y Territorio. Estudios Territoriales, Volumen XLVI, $n^{\circ} 180,253-275$.

de Manuel, E. \& López, J. (2017): Dinamizar la regeneración urbana desde la escala barrial: aprendizajes y transferencias del proyecto Barrios en Transición. Ciudades, 20, 21-44.

Diener, E. \& SuCH, E. (1997): Measuring quality of life: economic, social and subjetive indicators. Social Indicator Research, 40, 189-216.

DWIJENDRA, N. (2013): Quality of affordable housing projects by public and private developers in Indonesia: The case of Sarbagita Metropolitan Bali. Journal of Geography and Regional Planning, Vol. 6(3), 69-81.
EZquiAgA, J.M. (2011): Sueños de la razón: genealogía de los paradigmas para una teoria del planeamiento en el pensamiento de Fernando Terán. Ciudad y Territorio Estudios y Territoriales, n.160-170, 497-506.

Félix, R. \& Riondet-Costa, D. \& Palma-LimA, J. (2019): Modelo de avaliação de áreas urbanas para receber projetos integrados de revitalização e mobilidade sustentável. EURE, Volumen 45, $n^{\circ} 134,77-98$.

Fraser, J. \& Chaskin, R. \& Bauzin, J. (2013): Making Mixed-Income Neighborhoods Work for Low-Income Households. Cityscape, 15(2), 83-100.

Garcia-Hípola, M. \& Beltrán, M. (2011): Hibridacción y destrucción selectiva como estratagias propulsoras en centros históricos de Salamanca y Estocolmo. Ciudades, 14, 133-156.

GASTALDI, F. (2017): La regeneración urbana en Italia a partir de la reutilización del patrimonio público inmobiliario: un catálogo de herramientas y protocolos. Ciudades, 20, 155-178.

Gibson, F. \& Prathers, J. (1977): Does Anything Work? Evaluating Social Programs. Beverly Hills (EEUU): Sage Publications.

- \& Langsta, M. (1982): An Introduction to Urban Renewal. London (United Kindom): Hutchinson.

Grande, G. \& Secondini, P. \& Virgilio, G. (1999): Development of an explorative analysis for public choices with the aid of a GIS: the Rimini metropolitan area housing market case study. A demand side analysis. 2nd AGILE Conference on Geographic Information Science, (págs. 15-17). Roma (Italia).

GRIFTH, J. (1996): Gentrifcación: Perspective on the return to the central city. Journal of Planning Education and Research 11 (2), 241-255.

GuerRAND, R. (2004): Cinquante ans après l'appel de l'abbé pierre, une nouvelle crise du logement populaire en France. Ciudades, 8, 37-70.

Hernández, A., al. (2015): Recuperando la ciudad. Estrategia para el. Madrid (España): Instituto Juan de Herrera (IJH).

- al. (2014): Análisis de las políticas estatales y europeas de regeneración urbana y rehabilitación de barrios. Ciudad y Territorio. Estudios Territoriales, Volumen XLVI, $n^{\circ} 179,182-191$.

Hugony, C. \& RocA, J. (2008): Indicadores para la evaluación de las ciudades históricas. ACE - Arquitectura, Ciudad y Entorno, Año III, núm. 8, 219-238.

HuUb, P. \& BeCKers, P. (2012): Evaluating regeneration policies for rundown industrial sites in the Netherlands. European Real Estate Society (ERES): eres2012_269, 2151-2169. Obtenido de www.ideas. repec.org/p/arz/wpaper/eres2012_269.html

Jiménez, C. \& Molina, P. \& NicolÁs, O. (2017). Procedimientos innovadores de gestión y financiación en actuaciones de rehabilitación y regeneración urbana. Ciudades, 20, 89-110.

Jornet, S. \& Llop, C. \& PAstor, J. (2008). El Plan Especial de transformación urbana del barrio de La Mina, Barcelona. Premio Nacional de Urbanismo. Urban, $n^{\circ} 13,166-169$. 
LAWless, P. (2007). Continuing dilemmas for area based urban regeneration: evidence from the New Deal for Communities Programme in England. People, Place \& Policy Online: 1/1, 14-21.

Lawless, P., \& Brown, F. (1986). Urban Growth and Change in Britain. London (United Kingdom): Harper and Row.

LEAL, J. (2004). Segregation and social change in Madrid metropolitan region. The Greek Review of Social Research, 113, 81-104.

LeAL, J. \& SoRAndo, D. (2013). Rehabilitación urbana y cambio social en las grandes ciudades españolas. Monografías de la Revista Aragonesa de Administración Pública, XV, 205-236.

LefebVRe, H. (1969). El derecho a la ciudad. Barcelona (España): Península.

LEÓN, S. (2013). Indicadores de tercera generación para cuantificar la sustentabilidad urbana. ¿Avances o estancamiento? EURE, Volumen 39, $n^{\circ} 118,173-198$.

LIN, K.-L. (2010). Determining key ecological indicators for urban land consolidation. International Journal of Strategic Property Management, 14:2, 89-103.

Lo, C. \& FABER, B. (1997). Integration of Landsat Thematic Mapper and Census Data for Quality of Life Assessment. Remote Sens Environ, 62, 143-157.

Ludwig, J., Kling, J., Katz, L., Sanbonmatsu, L., Liebman, J., Duncan, G. \& Kessler, R. (2008). What Can We Learn about Neighbourhood Effects from the Moving to Opportunity Experiment? American Journal of Sociology, 114, 144-88.

MANERo, F. (2010): La participación ciudadana en la ordenación del territorio: posibilidades y limitaciones. Cuadernos geográficos, 47, 47-71.

MARIANI, G. (2010): Indicadores Urbanos y Ciudad. La Ciudad Viva. Obtenido de http://www.laciudadviva.org/blogs/?p=7336; http://www.laciudadviva.org/blogs/?p=8012 http://www.laciudadviva.org/blogs/?p=8393

MoNGIL, D. (2010). Intervención integral en barrios: conceptos, instrumentos y elementos de mejora. Ciudades, 13, 139-161.

Morandi, C. \& Pessina, G. \& Scavuzzo, L. (2010): Strumenti innovativi per la riqualificazione de quartieri residenziali in Italia: tre casi esemplari. Ciudades, 13, 103-122.

Moulaert, F. \& Parra, C. \& Swyngedow, E. (2014). Ciudades, barrios y gobernanza multiescalar en la Europa urbana. EURE, Volumen 40, n० 119, 5-24.

NABARRo, R. (1980): Inner City Partnerships An Assessment of the First Programmes. Town Planning Review, Vol. 51, No. 1, 25-38.

NAVARRO, C. (2015a): ¿Qué es la regeneración urbana? La idea y su práctica. En NAvARRo, C., Mejorar la ciudad transformando sus barrios. Regeneración Urbana en Andalucía (1990-2015) (págs. 15-22). Sevilla (España): Centro de Sociología y Políticas Locales - Universidad Pablo de Olavide.
- (2015b): La lógica de la regeneración urbana: gobernanza y redes de implementación. En NAVARRo, C., Mejorar la ciudad transformando sus barrios. Regeneración Urbana en Andalucía (1990-2015) (págs. 123-128). Sevilla (España): Publicado por el Centro de Sociología y Políticas Locales - Universidad Pablo de Olavide.

- (2015c): A modo de conclusiones: condiciones de partida, gobernanza y tipos de efectos. En NAVARRO C., Mejorar la ciudad transformando sus barrios. Regeneración Urbana en Andalucía (1990-2015) (págs. 123-128). Sevilla (España): Centro de Sociología y Políticas Locales - Universidad Pablo de Olavide.

— \& Rodríguez, M. (2015a): El diseño de los proyectos de regeneración urbana: la adaptación del "frame" y los "escenarios evaluativos". En Navarro, C., Mejorar la ciudad transformando sus barrios. Regeneración Urbana en Andalucía (1990-2015) (págs. 35-51). Sevilla (España): Centro de Sociología y Políticas Locales - Universidad Pablo de Olavide.

— \& Rodríguez, M. (2015b): Informe 4.2 Regeneración urbana y modelos de gobernanza: Estudio comparado de casos. Proyecto de I+D+i Regeneración Urbana y Cohesión Social en Andalucía (RUCOSA), Universidad Pablo de Olavide, Centro de Sociología y Políticas Locales, Sevilla (España).

- \& Echaves, A. \& MoyA, R. (2015a): Los proyectos de regeneración urbana y sus barrios: selección y caracterización de los casos. En NAVARRo, C., Mejorar la ciudad transformando sus barrios. Regeneración Urbana en Andalucía (1990-2015) (págs. 23-34). Sevilla (España): Centro de Sociología y Políticas Locales - Universidad Pablo de Olavide.

— \& Echaves, A. \& MoyA, R. (2015b): Informe 1.3 Los proyectos de regeneración urbana y sus barrios: selección y caracterización de los casos. Sevilla (España): Centro de Sociología y Políticas Locales - Universidad Pablo de Olavide.

- \& Echaves, A. \& Moya, R. \& Betanzos, J. (2015). Informe 2.1 ¿Ha cambiado el barrio? La delimitación de áreas experimentales y equivalentes. Proyecto de $\mathrm{I}+\mathrm{D}+\mathrm{i}$ Regeneración Urbana y Cohesión Social en Andalucía (RUCOSA), Universidad Pablo de Olavide, Centro de Sociología y Políticas Locales, Sevilla (España).

— \& Guerrero, G. \& Moya, R. (2015). Informe 2.2 Los impactos de la regeneración urbana: cambios temporales en áreas experimentales y equivalentes. Proyecto de I+D+i Regeneración Urbana y Cohesión Social en Andalucía (RUCOSA), Centro de Sociología y Políticas Locales-Universidad Pablo de Olavide, Sevilla (España).

— \& Rodriguez, M. \& Zapata, A. \& Mateos, C. (2015a): ¿Cómo han afectado los proyectos a los residentes? En Navarro, C., Mejorar la ciudad transformando sus barrios. Regeneración Urbana en Andalucía (1990-2015) (págs. 81-112). Sevilla (España): Centro de Sociología y Políticas Locales Universidad Pablo de Olavide. 
- (2015b): Informe 3 El impacto de los proyectos de regeneración urbana: el contexto a la exposición a las medidas de regeneración urbana. Proyecto de I+D+i Regeneración Urbana y Cohesión Social en Andalucía (RUCOSA), Centro de Sociología y Políticas Locales - Universidad Pablo de Olavide, Sevilla (España).

Organización de Cooperación y de Desarrollo Económico (OCDE) (1997): Un sistema de indicadores urbanos. Gabinete de Estudios y Estadísticas, Ayto. de Vitoria-Gasteiz (España).

OrganizACIÓN DE LAS NACIONES UNIDAS (ONU) (2015): Resolución 70/1 de la Asamblea General "Transformar nuestro mundo: la Agenda 2030 para el Desarrollo Sostenible" A/RES/70/1. Obtenido de https://www.un.org/ga/search/view doc. asp?symbol=A/70/L.1\&Lang=S

PARKINSON, M. (2014): Regeneración urbana integrada en 2014: nada nuevo bajo el sol y ¿Qué pasa con España? Ciudad y Territorio. Estudios Territoriales, Volumen XLVI, $n^{\circ} 179,11-27$.

Rhodes, J. \& Tyler, P. \& Brennan, A. (2005): Assessing the Effect of Area Based Initiatives on Local Area Outcomes: Some Thoughts Based on the National Evaluation of the Single Regeneration Budget in England. Urban Studies, 42 (11), 1919-1946.

Rosa, C. \& MÁrquez, M. \& NavAs, D. (2017). Hacia un nuevo modelo de gestión y autofinanciación de la regeneración de barriadas obsoletas. Ciudades, 20, 45-70.

Rubio, E. \& UREÑA, J.M. (2020): Metodología para evaluar la regeneración de barrios no planificados: el Barrio de San Antón en Cuenca. A: LLoP, C. \& Cenvera, M. \& Peremiquel, F. (eds.). "IV Congreso ISUF-H: Metrópolis en recomposición: prospectivas proyectuales en el Siglo XXI: Forma urbis y territorios metropolitanos, Barcelona, 28-30 Septiembre 2020". Barcelona: DUOT, UPC, 2020, p. 1-16.

RUEDA, S. (1999): Modelos e Indicadores para ciudades más sostenibles. Taller sobre indicadores de Huella y Calidad Ambiental Urbana. Fundación Fórum ambiental en colaboración con la Agencia Europea de Medio Ambiente.

Ruiz-Apilánez, B. \& Ureña, J. \& Solís, E. (2014): La revitalización de la calle: estrategias basadas en la remodelación. Ciudad y Territorio Estudios Territoriales, $n^{\circ} 181$, 393-412.

SAIZ, J. (2004): Los cambios en la vivienda de la exRepública Democrática Alemana a pafrtir de la reunificación. Ciudades, 8, 68-35.

Salas, J. \& Yepes, V. (2019a): A discursive, manyobjective approach for selecting more-evolved urban vulnerability assessment models. Journal of Cleaner Production (Capítulo 3). https://doi.org/10.1016/j.jclepro.2017.11.249

- (2019b): MS-ReRO and D-ROSE methods: assessing relational uncertainty and evaluating scenarios' risks and opportunities on multi-scale infrastructure systems. Journal of Cleaner Production. Vol. 216, 607-623. https://doi.org/10.1016/j.jclepro.2018.12.083
- (2019c): VisualUVAM: A Decision Support System Addressing the Curse of Dimensionality for the Multi-Scale Assessment of Urban Vulnerability in Spain. Sustainability 2019, 11, 2191. https://doi.org/10.3390/su11082191

SÁNCHEZ dE MAdARIAGA, I. (2004): Infraestructuras para la vida cotidiana y calidad de vida. Ciudades, 8 , 101-133.

Sánchez de Madariaga, I. \& García, J. \& Sistro, R. (2018): Mirando hacia el futuro: ciudades sostenibles. Los Objetivos de Desarrollo Sostenible en 100 ciudades españolas. Madrid (España): Red Española para el Desarrollo Sostenible (REDS).

Segado-VÁzquez, F. \& Espinosa-Muñoz, V. (2015): La ciudad herida. Siete ejemplos paradigmáticos de rehabilitación urbana en la segunda mitad del siglo xx. EURE, Volumen 41, $n^{\circ} 123,103-129$.

Serrano, E. \& al. (2014): Evaluación del impacto en salud de intervenciones de regeneración en la bahía de Pasaia: percepciones de la población afectada. Gaceta Sanitaria: 28(6), 442-449.

SHort, J. (1982): Housing in Britain: The Post-War Experience. London (United Kingdon): Methuen.

Simón-Rojo, M. \& Hernandez-Aja, A. (2011): Herramientas para evaluar la sostenibilidad de las intervenciones urbanas en barrios. Informes de la Construcción, Vol. 63, 41-49.

SMiтH, N. (1979): Toward a Theory of Gentrification A Back to the City Movement by Capital, not People, Journal of the American Planning Association, 45:4, 538-548.

Soles I Coll, G. (s.f.): El derecho a la ciudad en África Subsahariana. Obtenido de: https://www.africaye.org/derecho-ciudad-viviendaafrical

Solis, E., UReÑA, J. \& Mohíno, I. (2018): Centralidad territorial y especialización funcional como guía para la intervención en municipios con conjunto histórico. El caso de la región urbana madrileña. ACE: Architecture, City and Environment, 13 (37), 99-132.

TaYlor, D. \& Balloch, S. (2005): The politics of evaluation: an overview. En TAYLOR, D., \& BALLOCH, S. (Edits.), The politics of evaluation. Bristol, United Kingdom: The Polity Press.

Tello-Campos, C. \& Aguilar-Martinez, A. (2013): Regeneración y calidad de vida urbana en el centro antiguo de las ciudades de Montreal y México: una comparación. Economía, Sociedad y Territorio, vol. XIII, núm. 41, 121-149.

TroItIÑO, M. A. (1998): Turismo y desarrollo sostenible en ciudades históricas. Eria, 47, 211-227.

VARGAS-YÁÑEZ, A. (2015): Los indicadores de sostenibilidad de los municipios españoles: una propuesta metodológica a la vista de su evolución entre 2002-2015. Il International Congress on Sustainable Construction and Eco-Efficient Solutions. Sevilla (España).

Velázquez, I. \& Verdaguer, C. (2011): Regeneración urbana integral, tres experiencias europeas innovadoras: Île de Nantes, Coin Street y Barrio de la Mina. Madrid (España): SEPES Entidad Estatal de Suelo. 
Verdaguer, C. (2005): Evaluación del espacio público. Indicadores experimentales para la fase de proyecto. En Hernández, A., Proyecto en trabajo de investigación: Periferias, Sostenibilidad y Vitalidad Urbana. Madrid (España): Escuela Técnica Superior de Arquitectura de Madrid.
Von Breymann, H. (2014): La satisfacción residencial como herramienta de evaluación de proyectos de rehabilitación urbana. Ponencia presentada en el VI Seminario Internacional de Investigación en Urbanismo, Barcelona-Bogotá. Barcelona (España). 